\title{
Stage 0a Urethral Cancer AJCC v8
}

National Cancer Institute

\section{Source}

National Cancer Institute. Stage Oa Urethral Cancer A/CC v8. NCI Thesaurus. Code C140458.

Stage 0a includes: Ta, NO, MO. Ta: For male penile urethra and female urethra: Noninvasive papillary carcinoma. NO: No regional lymph node metastasis. M0: No distant metastasis. (AJCC 8th ed.) 